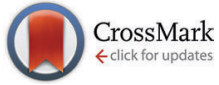

Cite this: Phys. Chem. Chem. Phys., 2015, 17, 14994

Received 9th March 2015 Accepted 9th May 2015

DOI: $10.1039 / c 5 c p 01371 b$

www.rsc.org/pccp

\title{
Photoluminescence of a single quantum emitter in a strongly inhomogeneous chemical environment $\dagger$
}

\author{
Anna M. Chizhik, ${ }^{a}$ Luigi Tarpani, ${ }^{\mathrm{b}}$ Loredana Latterini, ${ }^{\mathrm{b}}$ Ingo Gregor, ${ }^{\mathrm{a}}$ \\ Jörg Enderlein ${ }^{\star a}$ and Alexey I. Chizhik*a
}

\begin{abstract}
We present the results of a comprehensive photoluminescence study of defect centres in single $\mathrm{SiO}_{2}$ nanoparticles. We show that the photo-physical properties of the luminescent centres strongly resemble those of single dye molecules. However, these properties exhibit a large variability from particle to particle due to the different local chemical environment around each centre of each particle. This variability provides new insight into the complex photo-physics of single quantum emitters embedded into a random chemical environment. Moreover, a better understanding of the fundamental mechanism of the photoluminescence of defect centres in $\mathrm{SiO}_{2}$ structure is paramount for their application as white-light sources, non-toxic labels for bio-imaging, or for combining them with metallic and semiconductor nanostructures.
\end{abstract}

\section{Introduction}

One of the key parameters that determines the luminescence properties of any quantum emitter is its local chemical environment. Its inhomogeneity obliterates the distinctive features of an individual quantum emitter and broadens the distribution of its property values in an ensemble. It has been shown that individual fluorophores can exhibit a strongly heterogeneous distribution of their fluorescence quantum yield (QY) when embedded into a thin crystalline ${ }^{1}$ or amorphous ${ }^{2,3}$ dielectric matrix. In contrast to physical adsorption of fluorophores onto the surface or embedding them within a large homogeneous matrix, which is typically the case for the majority of single molecule fluorescence studies, the photo-physical properties of single quantum emitters that are chemically bound to a host matrix are poorly understood. At the same time, fundamental photo-physical aspects of interaction of a chemically bound chromophore with its local environment may provide a way

\footnotetext{
${ }^{a}$ III. Institute of Physics, Georg August University, 37077 Göttingen, Germany. E-mail: jenderl@gwdg.de, alexey.chizhik@phys.uni-goettingen.de

${ }^{b}$ Dipartimento di Chimica, Biologia e Biotecnologie, Università di Perugia and Centro di Eccellenza sui Materiali Innovativi Nanostrutturati, Via Elce di Sotto 8 , 06123 Perugia, Italy

$\dagger$ Electronic supplementary information (ESI) available: Synthesis of silica nanoparticles and size distribution analysis, photoluminescence measurements, generation of an azimuthally polarized laser beam, photoluminescence lifetime imaging of single $\mathrm{SiO}_{2}$ nanoparticles, the full series of single $\mathrm{SiO}_{2} \mathrm{NP}$ excitation spectra, the quantum yield of $\mathrm{SiO}_{2} \mathrm{NPs}$, photoluminescence decay curves of individual $\mathrm{SiO}_{2}$ nanoparticles, the histograms of the single particle lifetime and emission energy distributions, and the ensemble excitation spectrum of $\mathrm{SiO}_{2}$ nanoparticles. See DOI: 10.1039/c5cp01371b
}

towards easy and fast characterization of the surface chemistry for a wide range of applications in catalysis, photovoltaics, gas sensing, degradation of pollutants, and others. ${ }^{4-6}$

A typical example of an emitter that is chemically bound to a matrix is a luminescent centre in $\mathrm{SiO}_{2}$ nanoparticle (NP). ${ }^{7,8}$ It has been shown that the PL from $\mathrm{SiO}_{2}$ structures in the visible spectral range arises from non-bridging oxygen centres, ${ }^{9-11}$ neutral oxygen vacancies, ${ }^{11,12}$ and hydrogen-related species. ${ }^{13}$ Measurements of single $\mathrm{SiO}_{2}$ nanoparticle (NP) photoluminescence revealed a strong coupling between the electronic transition and collective vibrations in the $\mathrm{SiO}_{2}$ network due to a relaxation mechanism involving charge transfer. ${ }^{7,8}$ In particular, the PL spectra of a single $\mathrm{SiO}_{2}$ NP exhibit, besides a narrow zero-phonon line, one or even two satellite peaks at the lowerenergy end, which are related to the excitation of one or two longitudinal optical phonons in the particle. Furthermore, it has been shown that like single dye molecules, $\mathrm{SiO}_{2} \mathrm{NPs}$ possess both linear excitation and linear emission transition dipole moments. ${ }^{7}$ In contrast to luminescent centres in nanodiamonds that possess a regular crystal structure, defect centres in $\mathrm{SiO}_{2}$ NPs are embedded inside an amorphous matrix. ${ }^{14}$ This makes the defect centres a perfect model for studying photo-physical properties of individual dipole emitters within a highly inhomogeneous chemical environment. Moreover, unraveling the highly complex photo-physical properties of luminescent centres in $\mathrm{SiO}_{2}$ NPs will advance our understanding for using nanostructured $\mathrm{SiO}_{2}$ in electronics, ${ }^{15}$ drug delivery, ${ }^{16,17}$ synthesis of metallic ${ }^{18-21}$ and semiconductor ${ }^{22}$ nanoparticles with reduced toxicity, or for increasing their thermal stability and photoluminescence yield. ${ }^{23}$ 
Here, we present a comprehensive photoluminescence study of defect centres in single $\mathrm{SiO}_{2}$ NPs, which combines emission and excitation spectroscopy, fluorescence lifetime imaging, azimuthally polarized excitation dipole scanning, nanocavitybased QY measurements, and transmission electron microscopy. Because of the random variation of the local chemical environment around luminescent centres in $\mathrm{SiO}_{2}$ nanostructure, all the parameters of their photoluminescence are not readily accessible in ensemble measurements.

\section{Results and discussion}

For our experiments, we synthesized $\mathrm{SiO}_{2} \mathrm{NPs}$ of $11 \pm 1 \mathrm{~nm}$ diameter size by a modified Stöber method in a biphasic system using an amino acid as a base catalyst. ${ }^{24,25}$ Fig. 1(a) shows a transmission electron microscopy image of these NPs. For room temperature PL measurements, a droplet of $\mathrm{SiO}_{2}$ NPs in water suspension (Fig. 1(b)) was spin-coated on the surface of a glass cover slide. The particles were excited with an azimuthally polarized laser beam of $485 \mathrm{~nm}$ wavelength, focused into a diffraction-limited spot using a 1.49 numerical aperture objective lens. The azimuthal polarization of the excitation light

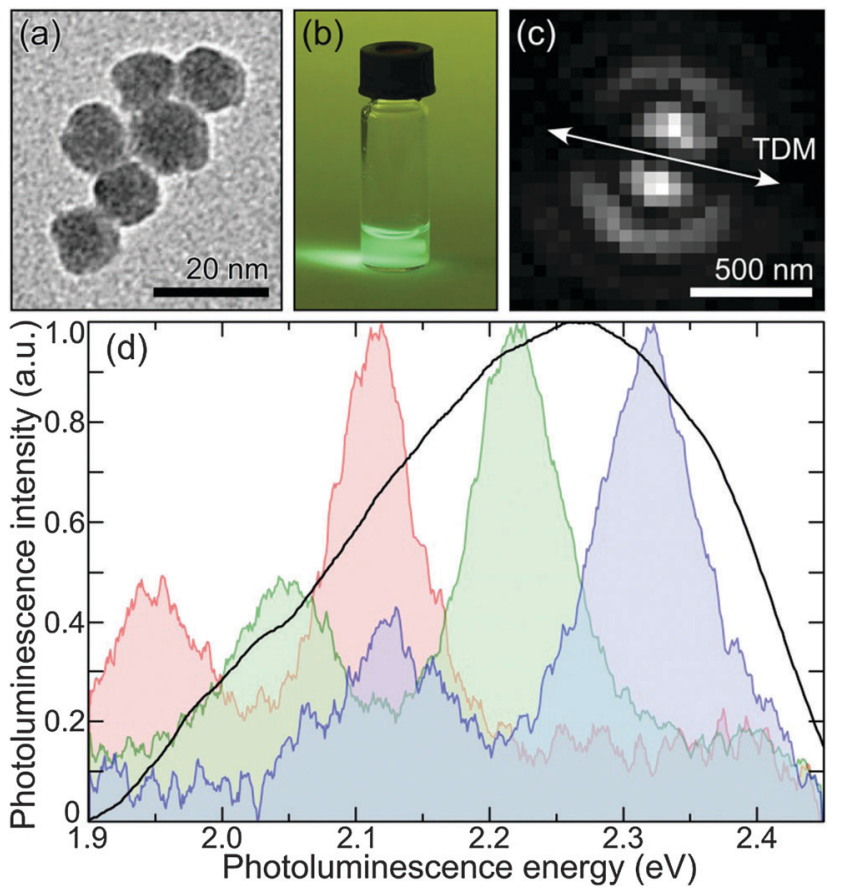

Fig. 1 (a) A transmission electron microscopy image of six $\mathrm{SiO}_{2}$ nanoparticles. (b) A photograph of a cuvette containing $\mathrm{SiO}_{2}$ nanoparticles in an aqueous solution upon excitation using a $485 \mathrm{~nm}$ laser. The photograph is taken through a $500 \mathrm{~nm}$ long-pass filter to block scattered laser light. (c) Excitation pattern of a single $\mathrm{SiO}_{2}$ nanoparticle when scanned with an azimuthally polarized laser focus. The arrow shows the projection of the excitation transition dipole moment on a sample plane. (d) Color-shaded area: three photoluminescence spectra of single $\mathrm{SiO}_{2}$ nanoparticles dispersed on the surface of a glass cover slide. Every spectrum exhibits different spectral shifts. Before plotting, the experimental data have been subjected to averaging over seven data points. Solid black line: the photoluminescence spectrum of the $\mathrm{SiO}_{2}$ nanoparticle ensemble. allowed us to verify that every measured $\mathrm{SiO}_{2} \mathrm{NP}$ behaves indeed as a single dipole emitter (Fig. 1(c)). ${ }^{26}$ Further details of the $\mathrm{SiO}_{2}$ NP synthesis and PL measurements can be found in the ESI. $\dagger$ Fig. 1(d) shows three examples of a single $\mathrm{SiO}_{2}$ particle's $\mathrm{PL}$ spectra together with an ensemble spectrum. The single particle spectra exhibit two narrow bands: a main emission maximum and a lower satellite at longer wavelength, which are attributed to the zero-phonon and phonon-assisted charge recombination on a defect centre, respectively. ${ }^{7}$ The striking similarity of the single particle spectra suggests that the observed PL originates from the defects with chemically identical structure, shifted by strong interaction with the inhomogeneous local environment. As a result of the individual shift of each of the single particle spectra, the two-band structure is smeared out in the ensemble measurement (black solid line in Fig. 1(d)).

In total, we recorded $87 \mathrm{PL}$ spectra of individual $\mathrm{SiO}_{2} \mathrm{NPs}$. The average full width at half-maximum (FWHM) for the zerophonon line is $80 \pm 5 \mathrm{meV}$. The separation between the zerophonon and phonon-assisted bands for all the measured single particle spectra is $170 \pm 20 \mathrm{meV}$, which can be attributed to the longitudinal optical mode of $\mathrm{SiO}_{2} \cdot{ }^{27}$ As the particles were not embedded into a polymer matrix during PL measurements, the emission energy and the phonon energy are solely related to the structure of the NPs and are not affected by any external local chemical environment. Moreover, in contrast to ref. 7, here, the particles were obtained not by oxidation of silicon nanocrystals, but initially synthesized as $\mathrm{SiO}_{2}$ nanostructure. This allows us to exclusively attribute the observed PL to the defect centres in $\mathrm{SiO}_{2}$ structure.

For each recorded emission spectrum, we determined the maximum emission wavelength and the peak intensity at this wavelength. Fig. 2(a) shows the resulting distribution for the measured 87 NPs. The homogenous, wavelength-unspecific dispersion of the emission intensity at each wavelength indicates the randomness of NP orientation, total extinction, and QY. The single particle spectroscopic measurements were combined with the FLIM imaging, which allowed us to determine single particle PL lifetimes. The single particle PL lifetime values were obtained by calculating an average photon arrival time, as it is typically the most reliable algorithm in the case of moderate fluorescence intensities. However, all the single particle decay curves exhibited a mono-exponential character, which confirms that they originate from a single quantum emitter. We show several typical examples of the single $\mathrm{SiO}_{2} \mathrm{NP}$ decay curves in Fig. S7 of the ESI. $\dagger$ Open circles in Fig. 2(b) show the distribution of PL lifetime versus intensity for the same $\mathrm{SiO}_{2}$ NPs as had been used for measuring single particle emission spectra. The histograms of the single particle lifetime and emission energy distributions are shown in Fig. S8 of the ESI. $\dagger$

At first we would like to discuss the results of single particle PL lifetime measurements. The distribution of the single particle PL lifetime versus intensity, which is shown in Fig. 2(b), allows us to estimate the QY value for all the measured $\mathrm{SiO}_{2} \mathrm{NPs}_{\text {. For }}$ this purpose we first measured the QY of an ensemble of NPs. The random spectral shift of single $\mathrm{SiO}_{2}$ NPs' emission makes it difficult to use standard methods for measuring their QY. 

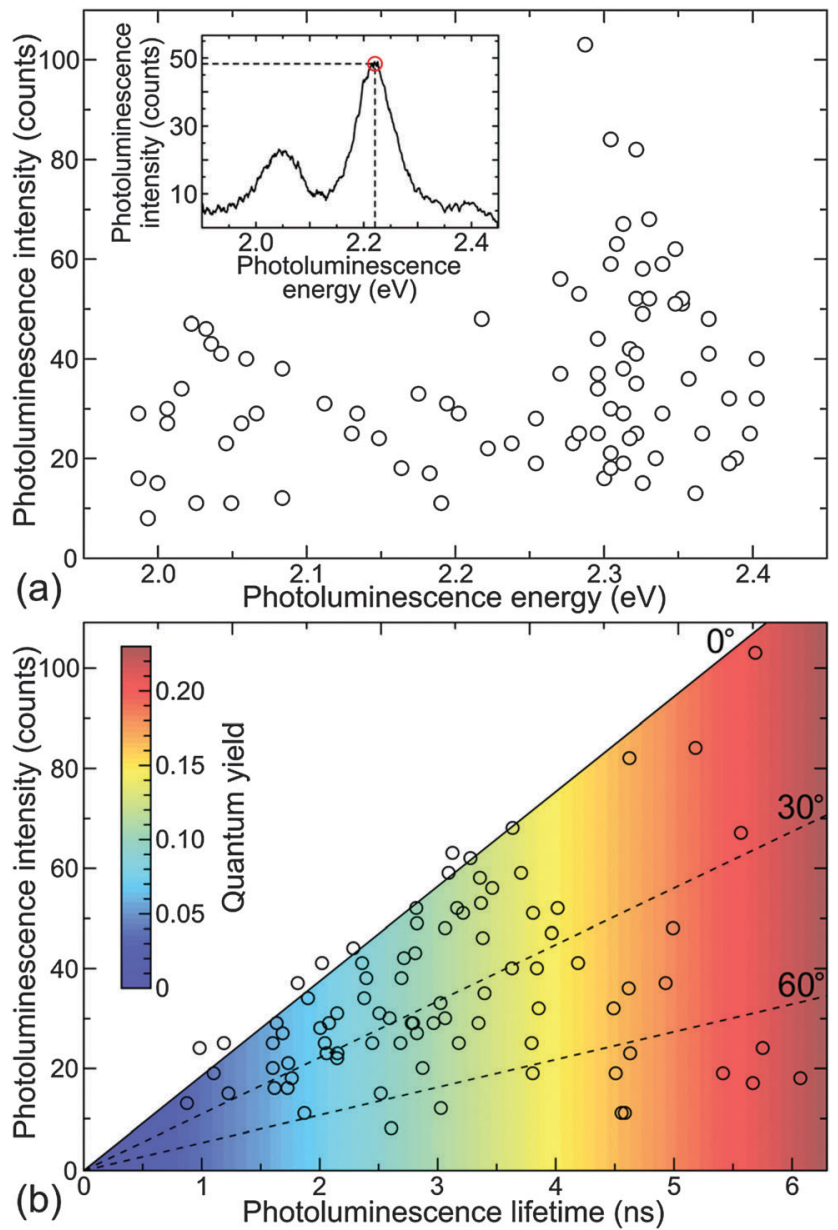

Fig. 2 (a) Zero-phonon line energy versus intensity for single $\mathrm{SiO}_{2}$ nanoparticle photoluminescence spectra. The inset shows one single emission spectrum and how the data (intensity at maximum emission wavelength versus maximum emission wavelength) for the main plot were determined. (b) Photoluminescence lifetime versus intensity for the same single $\mathrm{SiO}_{2}$ nanoparticles, which were used for the measurement of emission spectra. The color scale shows the distribution of the QY, which was obtained from the ensemble QY value and single particle lifetime distribution. The ensemble QY value was measured using the cavity-based method (see main text). The angle labels indicate the out-of-plane inclination of the particle's transition dipole.

However, our recently developed nanocavity-based method of QY determination measures only the cavity modulated radiative decay rate of an emitter, which makes it also applicable for complex systems such as $\mathrm{SiO}_{2} \mathrm{NPs}^{28,29}$ Placing $\mathrm{SiO}_{2}$ NPs between the metal mirrors of a nanocavity changes their emission behavior due to a cavity-modified electromagnetic field mode density. ${ }^{8,30,31}$ Because the cavity changes only the radiative rate of the embedded emitters, measuring the modulation of the PL lifetime as a function of the cavity length allows for determining an absolute value of an emitters' QY. ${ }^{32}$ Moreover, as the method is based on the cavityinduced lifetime modulation, it excludes the non-emitting (dark-state) species from the measurement. Thus, it provides the QY of only the optically active NPs.

Open circles in Fig. S6 of the ESI $\dagger$ show the results of $\mathrm{PL}$ lifetime measurements of $\mathrm{SiO}_{2}$ NPs in a droplet of water placed into a metallic nanocavity as a function of cavity length. The solid curve shows a fit of a theoretical model to the experimental data, where the free fit parameters are the QY value $\Phi$ and the free space PL lifetime $\tau_{0}$ (i.e., lifetime in aqueous solution without a cavity). The calculated free space lifetime value of $2.9 \mathrm{~ns}$ is in good agreement with the average free space lifetime measured for single $\mathrm{SiO}_{2}$ NPs (3.1 ns, see Fig. 2(b)). The QY of an emitter can be written as

$$
\Phi=\frac{k_{\mathrm{rad}}}{k_{\mathrm{rad}}+k_{\mathrm{nr}}}=\tau \cdot k_{\mathrm{rad}}
$$

where $k_{\mathrm{rad}}, k_{\mathrm{nr}}$, and $\tau$ are the radiative rate, non-radiative rate, and the PL lifetime of an emitter, respectively. As has been recently shown, the variation of the local chemical environment results in the change in the emitter's non-radiative transition rate, while leaving the radiative rate unaffected. ${ }^{2}$ Hence, the change in a single particle's QY with respect to the one measured in the ensemble will be proportional to the change in the particle's PL lifetime:

$$
\frac{\Phi}{\Phi_{\mathrm{av}}}=\frac{\tau}{\tau_{\mathrm{av}}}
$$

where index av indicates the average value measured on the ensemble. Eqn (2) allows us to translate the measured single $\mathrm{SiO}_{2}$ NP lifetime values (open circles in Fig. 2(b)) into QY values. The obtained QY distribution as shown with color shading in Fig. 2(b) indicates that the QY of the luminescent centres in $\mathrm{SiO}_{2}$ NPs has a broad distribution and can exceed 0.2.

Along with a change in the PL lifetime, the particles exhibit a strong variation of the emission intensity. The latter, however, is dependent on the particle's transition dipole orientation with respect to the excitation field polarization, and hence, cannot be used as an absolute parameter for QY estimation. The orientationdependent brightness of the particles manifests itself by the wedge-like shape of the distribution of the experimental data in Fig. 2(b) with a clearly defined upper edge, where intensity increases linearly with the lifetime (and hence, the QY). The upper edge of the plot (solid line) corresponds to the highest excitation efficiency of a particle, when its transition dipole lies within the horizontal plane. Because single particles were excited with an azimuthally polarized laser beam, which excites all particles with horizontally dipole orientation with the same efficiency, the decrease of the PL intensity is caused by an out-of-plane inclination of the particle's transition dipole. The dashed lines indicate the transition dipole inclination angles of $30^{\circ}$ and $60^{\circ}$ with respect to the horizontal plane.

Next, we discuss the results of the spectroscopic study. By employing the random nature of the spectral shifts of the $\mathrm{SiO}_{2}$ particles' PL, we reconstruct the shape of a single particle's excitation spectrum. Fig. 3 depicts the core principle used to reconstruct the excitation spectrum from emission spectra measurements. The two core initial assumptions are (i) that the shape of the excitation spectra and (ii) the Stokes shift are the same for all the emission centres observed. These assumptions are supported by the fact that despite varying spectral shift and 


\section{Emission spectrum}

\section{Excitation spectrum}

- - - Wavelength-dependent emission intensity

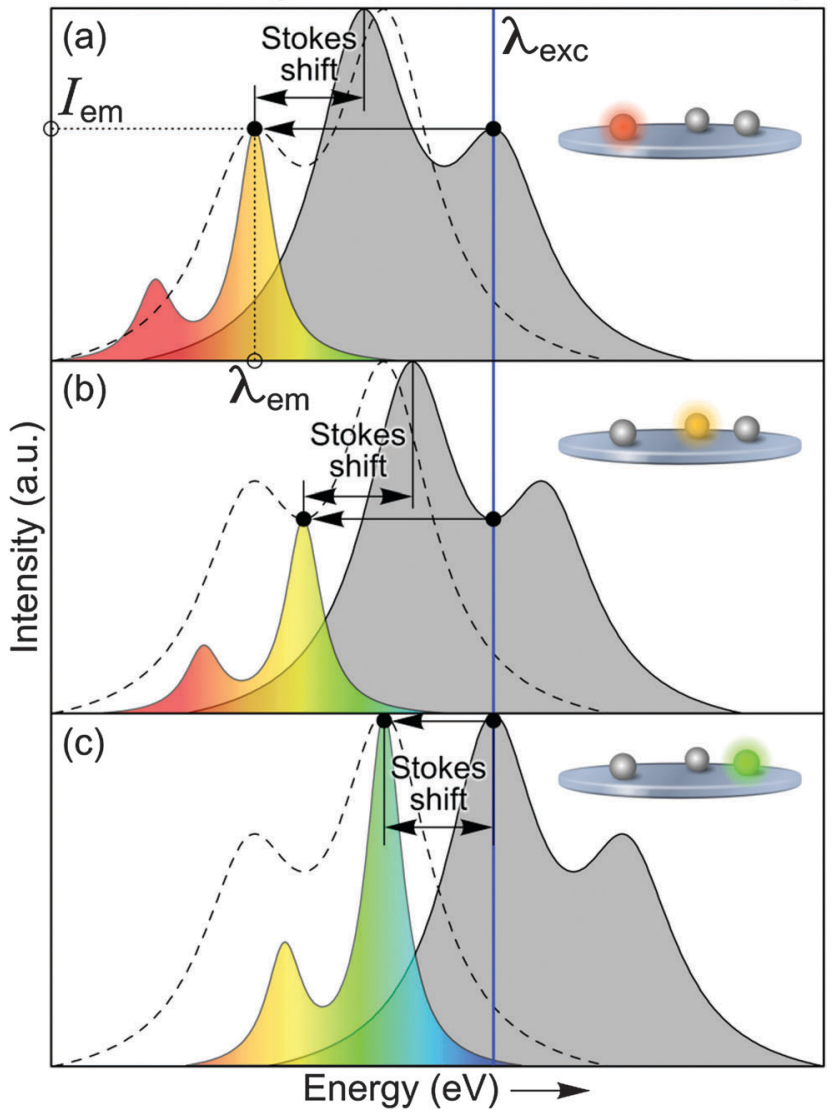

Fig. 3 Schematic of how an excitation spectrum is reconstructed from emission spectra measurements. The multi-color shaded areas show typical emission spectra of three single $\mathrm{SiO}_{2}$ nanoparticles. The grey shaded area represents a schematic of the single particle excitation spectrum. The Stokes shift is fixed for all the $\mathrm{SiO}_{2}$ nanoparticles. The dashed curve shows the modulation of the wavelength-dependent photoluminescence intensity maximum, which is determined by the shape of the excitation spectrum The vertical solid blue line indicates the excitation wavelength. Inset: schematic of the luminescent $\mathrm{SiO}_{2}$ particles on the surface of a glass cover slide.

emission intensity, the shape of the PL spectra of all the measured $\mathrm{SiO}_{2}$ NPs does not exhibit a noticeable variation.

If the excitation spectra as well as the excitation peculiarities (orientation of an excitation electric field vector relative to dipole orientation, emission QY, etc.) would be identical for all NPs, then a plot of the emission intensity at maximum emission wavelength $\left(I_{\mathrm{em}}\right)$ versus this maximum emission wavelength $\left(\lambda_{\mathrm{em}}\right)$ for different particles will trace the excitation spectrum of a single NP. The grey shaded area in Fig. 3(a)-(c) represents the single particle excitation spectrum, which is initially unknown. The dashed line in Fig. 3(a)-(c) shows the mirror image of the excitation spectrum. The three single particle emission spectra (color shaded areas in Fig. 3) demonstrate how the emission maxima are modulated at different spectral shifts, which allows for reconstructing the mirror image of the excitation spectrum.
Since the maximum of the reconstructed spectrum corresponds to the highest possible single particle emission intensity (Fig. 3(c)), the separation between the excitation energy and maximum of the reconstructed spectrum corresponds to the Stokes shift. In reality, each NP has a different orientation with respect to the exciting light polarization and QY of emission. However, these parameters are randomly distributed (Fig. 2(b)). Therefore, if the shape of the excitation spectra of all NPs is the same, then the envelope of the resulting histogram of the PL intensities versus maximum emission wavelength will be proportional to the excitation spectrum.

Fig. 4 shows a histogram of measured single NP PL intensity versus energy. The histogram exhibits two maxima, which can be well fitted with two Lorentzian functions (red curves in Fig. 3). This fit represents the reconstructed, horizontally inverted excitation spectrum of a single $\mathrm{SiO}_{2} \mathrm{NP}$, while the energy separation between its maximum and the excitation wavelength is the Stokes shift $(220 \mathrm{meV})$. The relatively large Stokes shift can be possibly attributed to the strong chemical binding of the luminescence centre to the surrounding $\mathrm{SiO}_{2}$ matrix, which can potentially strengthen vibrational dissipation of energy. Despite the similarity between the shapes of the $\mathrm{SiO}_{2} \mathrm{NP}$ emission spectra and the reconstructed excitation spectrum, the latter is broadened. In particular, the separation of the bands in the excitation spectrum is $280 \mathrm{meV}$ and their FWHM is $230 \mathrm{meV}$, against 170 and $80 \mathrm{meV}$ for the emission spectrum, respectively.

We attribute the observed broadening of the excitation spectrum to spectral diffusion, as commonly observed for single molecule spectra. ${ }^{33,34}$ This effect can be also related to a redistribution of defect states within a particle, which has been recently observed as a reversible flipping of the transition dipole moment, while the particle orientation itself was fixed. ${ }^{7}$ This can happen if another defect in a NP becomes energetically more favorable and then serves as the energy trap from which luminescence occurs. Similar cases of several co-existing conformations of a fluorophore

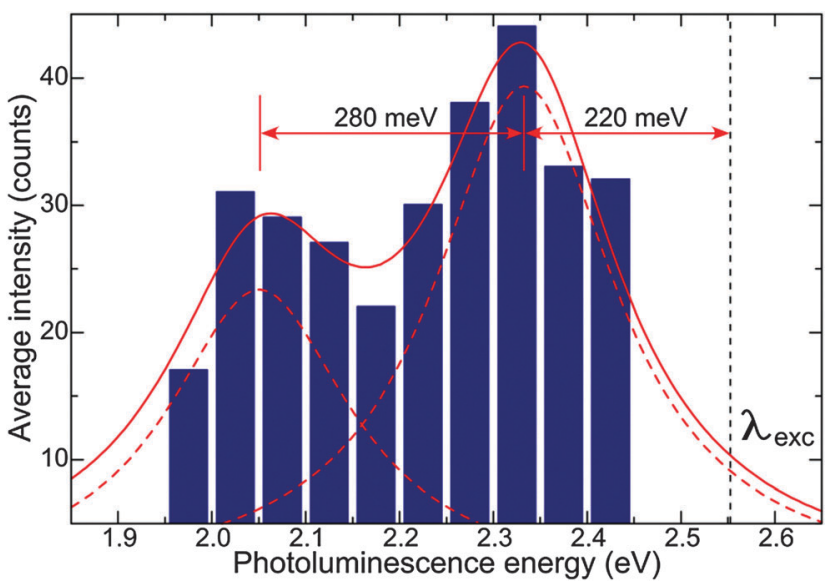

Fig. 4 Histogram of the photoluminescence intensity with a 50 meV bin width. The solid red curve shows a fit of two Lorentzian functions to the data. The dashed red curves show the individual Lorentzian peaks. The black dashed line represents the excitation laser energy. The energy separation between the main maximum and the laser energy yields the values of the Stokes shift. 
have also been observed for various fluorescent proteins, which typically results in a broadening of the excitation and absorption spectra. ${ }^{35}$ Alternatively, the observed spectral broadening can be attributed to a Frank-Condon type overlap between the ground and excited state with different curvatures.

The distribution of the emission maxima partitions into two groups at roughly 2.0-2.05 and 2.3-2.35 eV, as has been observed in recent studies on luminescent defects in $\mathrm{SiO}_{2}$ nanoparticles and $\mathrm{Si} / \mathrm{SiO}_{2}$ core-shell nanocrystals. ${ }^{7,41,42}$ There, several controversial hypotheses have been proposed. In particular, different mechanisms of the exciton recombination in a $\mathrm{Si} / \mathrm{SiO}_{2}$ nanocrystal, or emission of a photon from different types of defects in a $\mathrm{SiO}_{2}$ structure was discussed.

To determine whether the measured distribution of the emission maxima is related to the shape of the excitation spectrum of a single defect, or to the emission from different luminescent centres, we measured excitation spectra of single particles directly, by scanning the excitation wavelength from 465 to $561 \mathrm{~nm}$. The emission was recorded within a spectral range of 570-615 nm, limited by a band pass filter. The measured PL intensity was normalized by the laser power at each excitation wavelength. The measurements were combined with the FLIM images, which allowed us to determine the single particle's PL lifetime. Fig. S4 (ESI $\dagger$ ) shows examples of the collected PL intensity and lifetime images of the same sample area, containing two optically active $\mathrm{SiO}_{2}$ NPs, where we have used different excitation wavelengths from 488 to $530 \mathrm{~nm}(2.53-2.34 \mathrm{eV})$. Whereas the emission intensity exhibits a strong dependence on excitation wavelength, the PL lifetime does not show any noticeable variation. Constant lifetime values were observed for each of the measured $\mathrm{SiO}_{2}$ NPs within the whole range of excitation wavelengths. Taking into account a broad variation of the PL lifetime values of different luminescent centres in $\mathrm{SiO}_{2}$ structure from 1 to 6 ns (see Fig. 2(b)), this suggests that the observed modulation of the PL intensity is solely related to the specific shape of the excitation spectrum, but not to the transition of the PL between different types of optically active defects.

Fig. S5 of the ESI $\dagger$ shows the excitation spectra of 7 single $\mathrm{SiO}_{2}$ NPs. The measured single particle excitation spectra were arbitrarily shifted so that their maxima coincided. Solid circles in Fig. 5 show the average spectrum, obtained from the excitation spectra of 7 single $\mathrm{SiO}_{2}$ NPs. By fitting the obtained curve with two Lorentzian functions we determined a band separation of $260 \mathrm{meV}$, which is in very good agreement with the value obtained using the excitation spectrum reconstruction. The ratio of the intensities for the two spectral bands is 0.7 , while it was 0.6 for the reconstructed spectrum.

To verify the results of the single particle study, we measured the ensemble excitation spectrum of $\mathrm{SiO}_{2}$ NPs in aqueous solution. The PL was recorded from the narrow spectral window of $2.25 \pm 0.01 \mathrm{eV}$, where the maximum emission intensity was observed (see Fig. 1(d)). The experimental curve exhibits two bands, however the lower one smeared out by averaging of the signal among the relatively large amount of particles. The Stokes shift is in excellent agreement with the value, which was obtained from the single particle study.

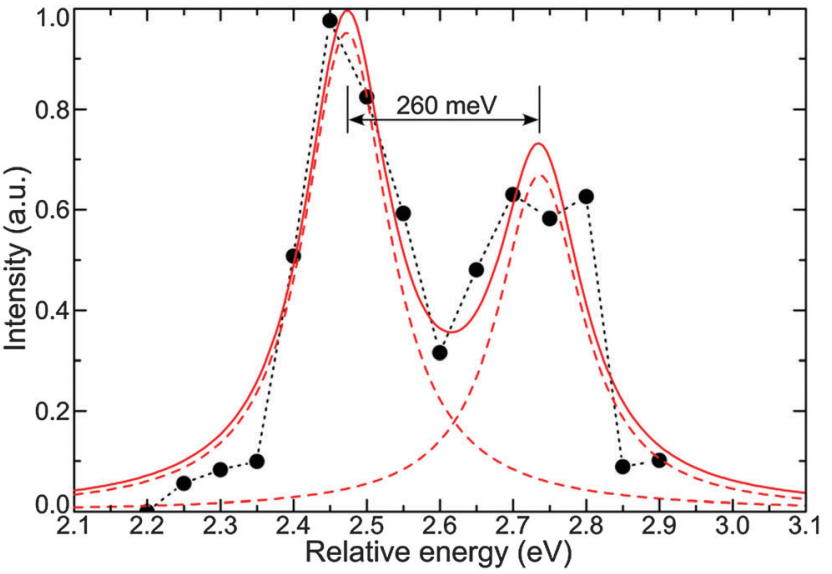

Fig. 5 Dotted line with solid circles: the excitation spectrum obtained as a result of averaging over 7 single $\mathrm{SiO}_{2}$ nanoparticle excitation spectra. Arbitrary shift of the single particle spectra is indicated by a relative energy axis. The solid red curve shows a fit of two Lorentzian functions to the data. The dashed red curves show the two individual Lorentzian peaks.

The obtained distribution of the maximum emission wavelength allows us to discuss the possible origin of the PL observed in the current work. According to the work of Glinka et al., ${ }^{13}$ the PL extending from 1.8 to $2.8 \mathrm{eV}$ with maximum at near $2.35 \mathrm{eV}$, which has been observed for thermally untreated $\mathrm{SiO}_{2}$ NPs with diameters from 7 to $15 \mathrm{~nm}$, can be attributed to hydrogen-related species ( $\equiv \mathrm{Si}-\mathrm{H}$ and $\equiv \mathrm{Si}-\mathrm{OH}$ ) on the surface of the nanoparticles. However, this model cannot explain the growth of the defect-related PL in $\mathrm{Si} / \mathrm{SiO}_{2}$ core-shell nanoparticles after the dehydrogenation of the sample. ${ }^{22}$ The observed spectral distribution of the PL from 2.0 to $2.4 \mathrm{eV}$ partly overlaps with a broad emission at $2.7 \mathrm{eV}^{36,37}(\mathrm{FWHM}=$ $0.8 \mathrm{eV}$ ), which has been attributed to neutral oxygen vacancy defects $(\equiv \mathrm{Si}-\mathrm{Si} \equiv){ }^{11,12}$ On the other hand, the red side of the distribution corresponds to an isolated non-bridging oxygen atom ( $\left.\equiv \mathrm{Si}-\mathrm{O}^{\bullet}\right)$ that acts as a hole-trap centre, ${ }^{9-11}$ which leads to the emission at near 1.9-2.0 eV. The overlap of the observed spectral distribution of the PL with all the three ranges, which correspond to the possible types of defects, makes it hard to exclusively attribute the observed PL to one of them. Attribution of the single particle PL to a particular type of a luminescent centre is additionally complicated by the possible presence of several types of defects within one nanoparticle, while only one of them is optically active. This is confirmed by the recent observation of the single particle transition dipole moment reorientation because of the redistribution of defect states within a particle. ${ }^{7}$ The influence of a hydrogen and oxygen atmosphere on the single $\mathrm{SiO}_{2}$ particle $\mathrm{PL}$ can potentially help to identify the origin of the single $\mathrm{SiO}_{2}$ particle PL. ${ }^{22,38,39}$ To obtain more detailed information on the possible attribution of the observed PL to a particular type of luminescent centre, dedicated studies will be carried out in the near future.

\section{Conclusions}

The results of the comprehensive PL study show that the photophysical properties of the luminescent centres in $\mathrm{SiO}_{2}$ 
nanoparticles partly resemble those of typical single dye molecules, for instance, linear transition dipole, symmetric emission and excitation spectra. However, a highly inhomogeneous local chemical environment around the centres results in a broad and random variation of the single NP emission and excitation spectra, PL lifetime, and QY. In particular, the PL lifetime and QY of different NPs can vary by a factor of 6, while both the emission and excitation spectra of different luminescent centres can exhibit a shift up to $500 \mathrm{meV}$. It is remarkable that despite a broad variation of the shift in both of the emission and excitation single particle spectra, the shape of the spectrum remains constant. The obtained results show the striking difference between the cases, when the photo-physical properties of a single quantum emitter are tailored by the local chemical environment or by the mode density of the electromagnetic field. ${ }^{31}$ In the latter case, the change in the field mode structure results in the redistribution of the emission spectrum and modification of the radiative rate of a chromophore, while the non-radiative rate remains constant. ${ }^{40}$ These findings provide new insight into the complex photo-physical properties of a single quantum emitter embedded within a highly inhomogeneous chemical environment. The presented results are of particular importance for designing $\mathrm{Si} / \mathrm{SiO}_{2}$ nanostructures with controllable resonance energy transfer between the quantum confined exciton in the crystalline core and luminescent centres in the silica shell. ${ }^{22,41,42}$

\section{Acknowledgements}

Funding by the German Science Foundation (DFG, SFB 937, project A5) is gratefully acknowledged. A.M.C. thanks the Dorothea Schlözer Fellowship Programme for financial support. L.L. thanks the support of the University of Perugia. L.T. acknowledges the support of Regione Umbria under the framework POR-FSE 2007-2013. We thank the reviewers of the manuscript for enhancing the quality of this work.

\section{Notes and references}

1 B. C. Buchler, T. Kalkbrenner, C. Hettich and V. Sandoghdar, Phys. Rev. Lett., 2005, 95, 063003.

2 A. I. Chizhik, A. M. Chizhik, D. Khoptyar, S. Bär, A. J. Meixner and J. Enderlein, Nano Lett., 2011, 11, 1700-1703.

3 Y. Cesa, C. Blum, J. M. van den Broek, A. P. Mosk, W. L. Vos and V. Subramaniam, Phys. Chem. Chem. Phys., 2009, 11, 2525-2531.

4 C. Lun Pang, R. Lindsay and G. Thornton, Chem. Soc. Rev., 2008, 37, 2328-2353.

5 M. Gratzel, Nature, 2001, 414, 338-344.

6 R. Selvaggi, L. Tarpani, A. Santuari, S. Giovagnoli and L. Latterini, Appl. Catal., B, 2015, 168-169, 363-369.

7 A. M. Chizhik, A. I. Chizhik, R. Gutbrod, A. J. Meixner, T. Schmidt, J. Sommerfeld and F. Huisken, Nano Lett., 2009, 9, 3239-3244.
8 A. I. Chizhik, A. M. Chizhik, A. M. Kern, T. Schmidt, K. Potrick, F. Huisken and A. J. Meixner, Phys. Rev. Lett., 2012, 109, 223902.

9 L. Skuja, J. Non-Cryst. Solids, 1998, 239, 16-48.

10 S. Munekuni, T. Yamanaka, Y. Shimogaichi, R. Tohmon, Y. Ohki, K. Nagasawa and Y. Hama, J. Appl. Phys., 1990, 68, 1212-1217.

11 E. P. O'Reilly and J. Robertson, Phys. Rev. B: Condens. Matter Mater. Phys., 1983, 27, 3780-3795.

12 R. Tohmon, Y. Shimogaichi, H. Mizuno, Y. Ohki, K. Nagasawa and Y. Hama, Phys. Rev. Lett., 1989, 62, 1388-1391.

13 Y. D. Glinka, S.-H. Lin and Y.-T. Chen, Appl. Phys. Lett., 1999, 75, 778-780.

14 A. Gruber, A. Dräbenstedt, C. Tietz, L. Fleury, J. Wrachtrup and C. v. Borczyskowski, Science, 1997, 276, 2012-2014.

15 D. A. Muller, T. Sorsch, S. Moccio, F. H. Baumann, K. EvansLutterodt and G. Timp, Nature, 1999, 399, 758-761.

16 M. A. Malfatti, H. A. Palko, E. A. Kuhn and K. W. Turteltaub, Nano Lett., 2012, 12, 5532-5538.

17 T. M. Shaffer, M. A. Wall, S. Harmsen, V. A. Longo, C. M. Drain, M. F. Kircher and J. Grimm, Nano Lett., 2015, 15, 864-868.

18 E. Prodan, C. Radloff, N. J. Halas and P. Nordlander, Science, 2003, 302, 419-422.

19 L. Latterini and L. Tarpani, J. Phys. Chem. C, 2011, 115, 21098-21104.

20 E. Lukianova-Hleb, Y. Hu, L. Latterini, L. Tarpani, S. Lee, R. A. Drezek, J. H. Hafner and D. O. Lapotko, ACS Nano, 2010, 4, 2109-2123.

21 L. Tarpani and L. Latterini, Photochem. Photobiol. Sci., 2014, 13, 884-890.

22 S. Godefroo, M. Hayne, M. Jivanescu, A. Stesmans, M. Zacharias, O. I. Lebedev, G. Van Tendeloo and V. V. Moshchalkov, Nat. Nanotechnol., 2008, 3, 174-178.

23 N. J. Halas, ACS Nano, 2008, 2, 179-183.

24 T. Yokoi, Y. Sakamoto, O. Terasaki, Y. Kubota, T. Okubo and T. Tatsumi, J. Am. Chem. Soc., 2006, 128, 13664-13665.

25 K. D. Hartlen, A. P. T. Athanasopoulos and V. Kitaev, Langmuir, 2008, 24, 1714-1720.

26 A. I. Chizhik, A. M. Chizhik, D. Khoptyar, S. Bär and A. J. Meixner, Nano Lett., 2011, 11, 1131-1135.

27 C. T. Kirk, Phys. Rev. B: Condens. Matter Mater. Phys., 1988, 38, 1255-1273.

28 A. I. Chizhik, I. Gregor and J. Enderlein, Nano Lett., 2013, 13, 1348-1351.

29 N. Karedla, J. Enderlein, I. Gregor and A. I. Chizhik, J. Phys. Chem. Lett., 2014, 5, 1198-1202.

30 A. I. Chizhik, I. Gregor, F. Schleifenbaum, C. B. Müller, C. Röling, A. J. Meixner and J. Enderlein, Phys. Rev. Lett., 2012, 108, 163002.

31 P. Goy, J. M. Raimond, M. Gross and S. Haroche, Phys. Rev. Lett., 1983, 50, 1903-1906.

32 A. I. Chizhik, I. Gregor, B. Ernst and J. Enderlein, ChemPhysChem, 2013, 14, 505-513.

33 W. P. Ambrose and W. E. Moerner, Nature, 1991, 349, 225-227. 
34 H. P. Lu and X. S. Xie, Nature, 1997, 385, 143-146.

35 R. E. Campbell, O. Tour, A. E. Palmer, P. A. Steinbach, G. S. Baird, D. A. Zacharias and R. Y. Tsien, Proc. Natl. Acad. Sci. U. S. A., 2002, 99, 7877-7882.

36 L. Rebohle, J. von Borany, H. Fröb and W. Skorupa, Appl. Phys. B: Lasers Opt., 2000, 71, 131-151.

37 G. Vaccaro, S. Agnello, G. Buscarino, M. Cannas and L. Vaccaro, J. Non-Cryst. Solids, 2011, 357, 1941-1944.

38 W. G. J. H. M. van Sark, P. L. T. M. Frederix, D. J. Van den Heuvel, H. C. Gerritsen, A. A. Bol, J. N. J. van Lingen, C. de Mello Donegá and A. Meijerink, J. Phys. Chem. B, 2001, 105, 8182-8284.
39 W. G. J. H. M. van Sark, P. L. T. M. Frederix, A. A. Bol, H. C. Gerritsen and A. Meijerink, ChemPhysChem, 2002, 3, 871-879.

40 A. Chizhik, F. Schleifenbaum, R. Gutbrod, A. Chizhik, D. Khoptyar, A. J. Meixner and J. Enderlein, Phys. Rev. Lett., 2009, 102, 073002.

41 T. Schmidt, A. I. Chizhik, A. M. Chizhik, K. Potrick, A. J. Meixner and F. Huisken, Phys. Rev. B: Condens. Matter Mater. Phys., 2012, 86, 125302.

42 J. Martin, F. Cichos, F. Huisken and C. von Borczyskowski, Nano Lett., 2008, 8, 656-660. 